Case Report and Review of the Literature

\title{
Supratentorial Hemangioblastoma in a Filipino Male with Von-Hippel Lindau: A Case Report and Systematic Review
}

\author{
Erin Camille A. Caritativo, Jo-Celine M. Leong*, Ma. Socorro C. Martinez, Jay B. Villavicencio and Manuel M. \\ Mariano
}

Institute for Neurosciences, St. Luke's Medical Center-Global, Taguig, Philippines

\begin{tabular}{l} 
A R T I C L E I N F O \\
\hline Article history: \\
Received: 30 September, 2021 \\
Accepted: 16 October, 2021 \\
Published: 4 November, 2021 \\
\hline Keywords: \\
Von Hippel-Lindau syndrome \\
supratentorial hemangioblastoma \\
renal cell carcinoma
\end{tabular}

\begin{abstract}
A B S T R A C T
Von Hippel-Lindau syndrome is a rare genetic condition characterized by the development of benign and malignant tumors in various organ systems. We report a case of a 58-year-old Filipino male with unremarkable family history who presented with progressive right homonymous hemianopia. Work up revealed presence of retinal and CNS hemangioblastomas as well as renal cell carcinoma. Confirmatory gene testing is pending but is ultimately not required to make the diagnosis.
\end{abstract}

(C) 2021 Jo-Celine M. Leong. Hosting by Science Repository.

\section{Introduction}

Von Hippel-Lindau disease (vHL) is a rare autosomal dominant genetic disorder characterized by predisposition to develop benign and malignant tumors in multiple organ systems such as the central nervous system (CNS), kidneys, adrenals, pancreas and reproductive organs. It is estimated to affect 1 in 36,000 people with about 200,000 cases reported worldwide [1]. However, due to the rarity of the condition and the relative difficulty in recognizing the syndrome, its incidence across the globe still vary widely. In the Philippines, the prevalence of vHL remains unclear and only a handful of reported cases exist.

\section{Case Report}

This is a case of a 58-year-old male who came in with 2-month history of progressive limitation of bilateral right visual field. Intermittent episodes of left-sided parietal headache were also reported but with no accompanying nausea, vomiting, focal weakness, sensory deficits or gait abnormalities. Review of systems was unremarkable. He denied any significant prior medical or surgical history including recent head trauma. There was no known familial history of malignant and benign tumors, blood dyscrasias, and neurologic disorders.

Upon consultation with an ophthalmologist, visual perimetry tes revealed right homonymous hemianopia. Plain cranial magnetic resonance imaging (MRI) showed an irregularly shaped focus at the left parietooccipital lobe that was hyperintense in $\mathrm{T} 1$ and $\mathrm{T} 2$ weighted images and exhibited marked susceptibility artifacts on susceptibilityweighted imaging (SWI). There were also noted T2W/FLAIR hyperintense and $\mathrm{T} 1$ isointense foci at the left superior parietal and right occipital lobes (Figure 1). At that time, there was consideration of tumoral hemorrhage versus vascular malformation. The patient was admitted for a week at a hospital in South Luzon and managed medically. He was discharged stable with residual right homonymous hemianopia.

${ }^{*}$ Correspondence to: Jo-Celine M. Leong, M.D., Neurosurgery Resident, Institute for Neurosciences, St. Luke's Medical Center-Global, Taguig, Philippines; Tel: +639175847362; E-mail: jo.celineleong@gmail.com

(C) 2021 Jo-Celine M. Leong. This is an open-access article distributed under the terms of the Creative Commons Attribution License, which permits unrestricted use, distribution, and reproduction in any medium, provided the original author and source are credited. Hosting by Science Repository. http://dx.doi.org/10.31487/j.SCR.2021.11.01 

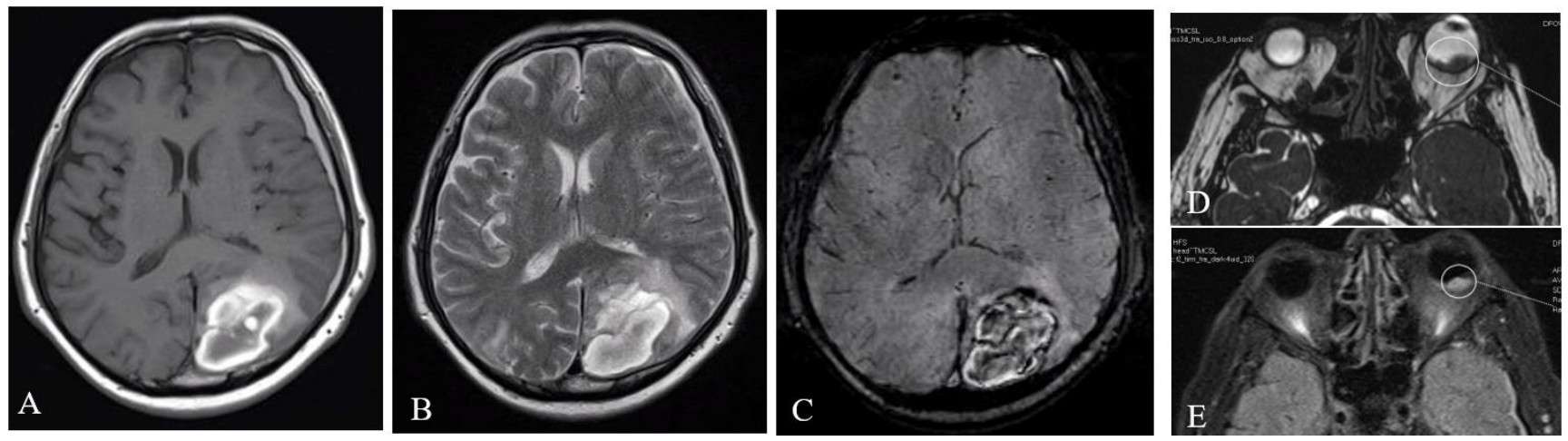

Figure 1: A \& B) Axial cuts of cranial MRI without contrast showing a T1 and T2W hyperintensity at the left parietooccipital lobe. C) It had corresponding susceptibility on SWI which was consistent with subacute intracerebral hemorrhage. D \& E) There is also an irregularly shaped focus that is isointense on T2W/FLAIR at the left subretinal area.

Upon consultation at our institution for further work up, the patient was noted to have episodes of blood pressure elevations. Plasma metanephrine and urine vanillylmandelic acid levels to rule out pheochromocytoma were within normal ranges. Cerebral angiogram was done showing several intracranial masses at the regions of right posterior parietal lobe, left medial occipital lobe and left posterior ocular orbit (Figure 2). Fluorescein angiography likewise showed sub-retinal mass in both eyes (Figure 3). Whole abdominal ultrasound was then requested for screening. It detected a large mass at the upper half of the right kidney and a small cyst at the left kidney. There was no pancreatic mass or adrenal lesion seen. Subsequent contrast-enhanced computed tomography (CT) urogram further delineated the lobulated heterogeneously-enhancing right renal mass as having malignant features (Figure 4A). In addition, high resolution chest CT scan revealed multiple varisized nodules in both lungs considering a metastatic process (Figure 4B).
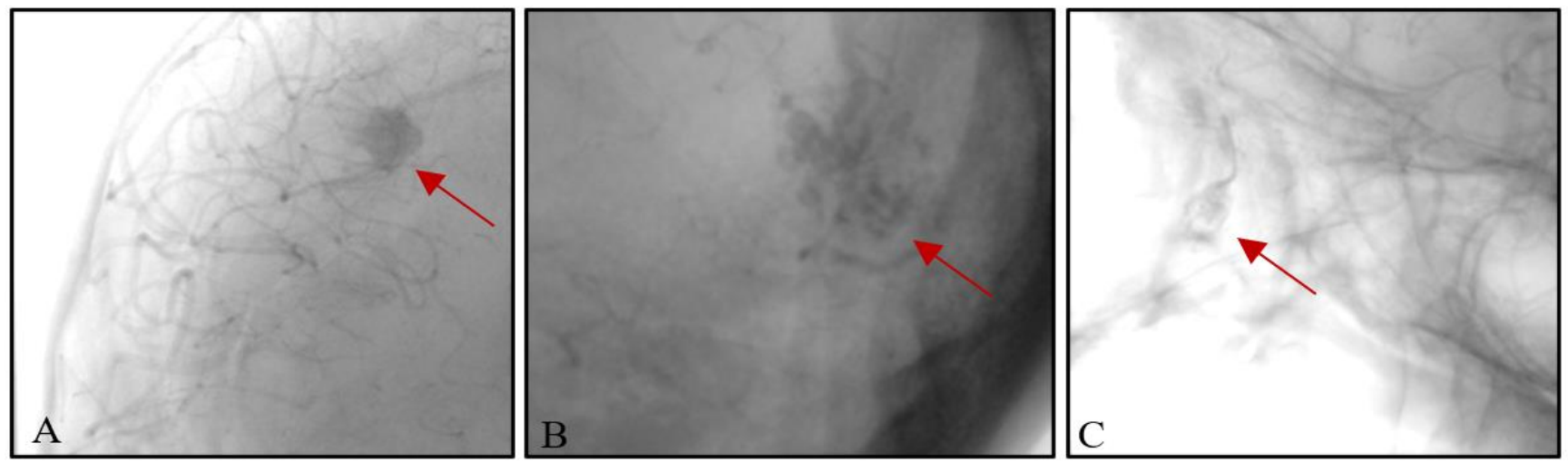

Figure 2: Cerebral angiogram revealing multiple intracranial and subretinal masses (red arrows). A) Right ICA injection (AP view) shows a tumoral blush on the right posterior parietal area. On left ICA injection (lateral view), there is noted tumoral blush in the B) left medial occipital region and C) left posterior orbit.

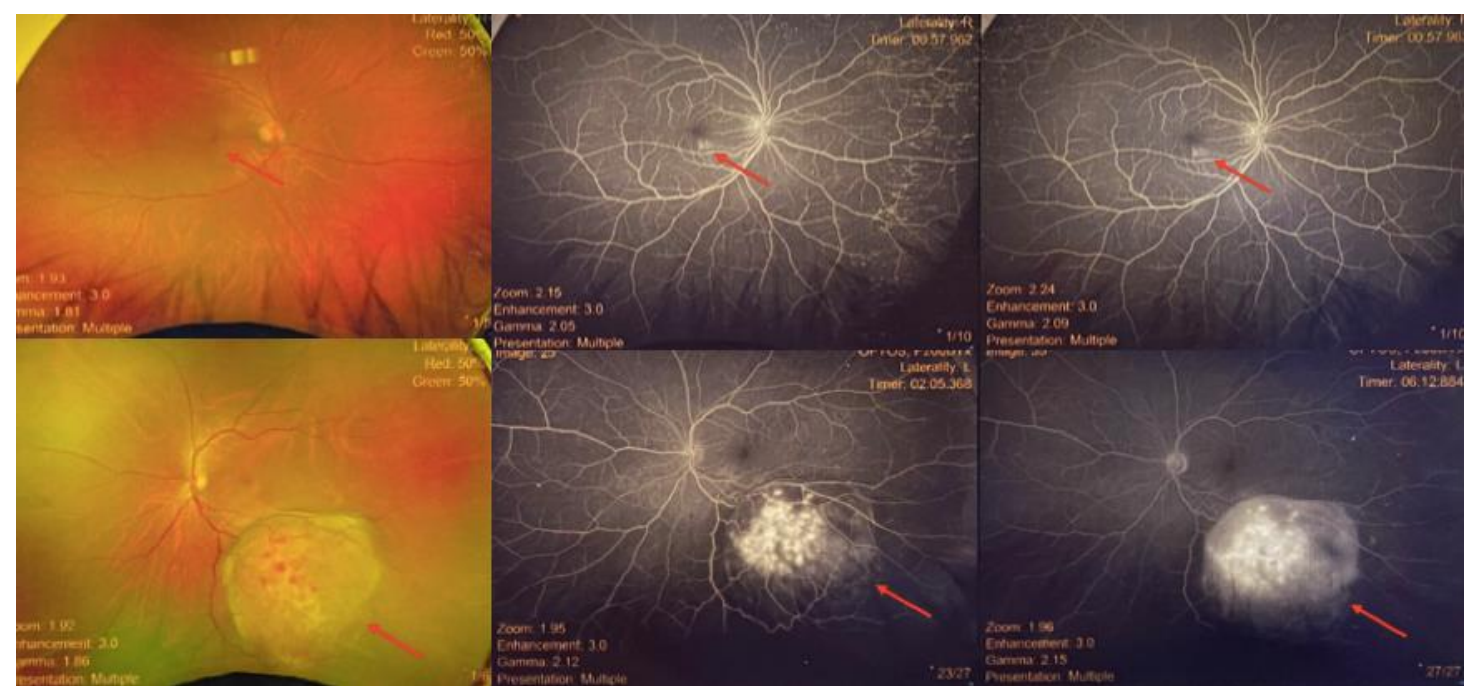

Figure 3: Fundus photo and fluorescein angiography showing bilateral subretinal mass (top row - right eye; bottom row - left eye). 


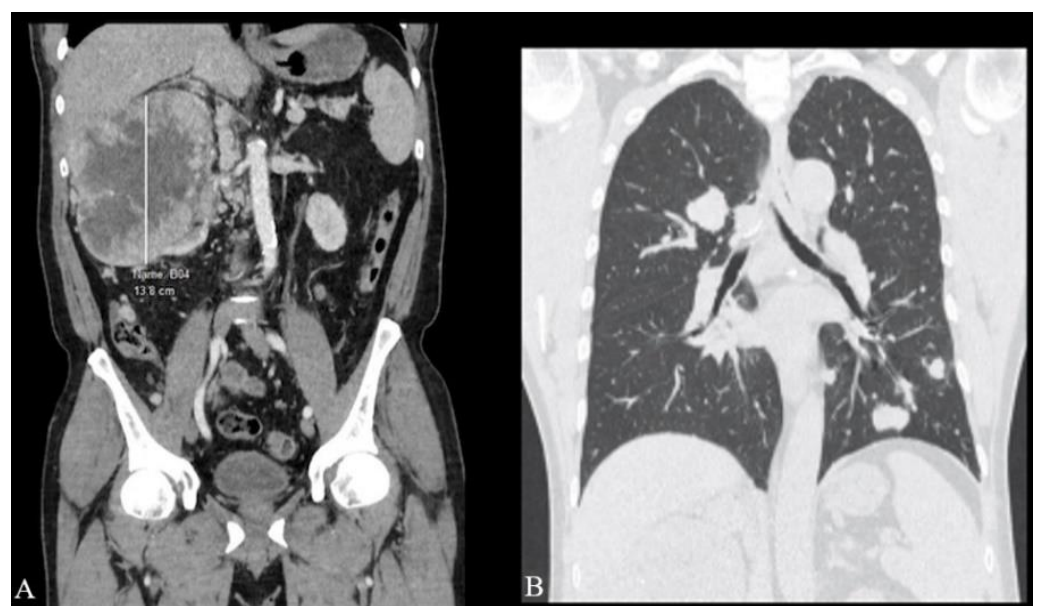

Figure 4: A) Axial cut of a contrast-enhanced computed tomography urogram revealing lobulated heterogeneously enhancing mass at right kidney. B) Axial cut of a high-resolution chest CT scan showing multiple nodules in both lungs.

The patient underwent open radical right nephrectomy with no adverse perioperative events. Histopathologic analysis showed clear cell renal cell carcinoma, WHO Grade 2. He was discharged stable with plans to undergo genetic testing as outpatient and to undergo oral chemotherapy.

\section{Discussion}

A search of PubMed, Cochrane, Google Scholar and Philippine journals such as Acta Medica Philippina and Philippine Journal of Science of the following MeSH terms ((Filipino or Philippines) AND (supratentorial) AND (hemangioblastoma) AND ((VHL or Von-Hippel Lindau) yielded no results (Figure 5). To the best of our knowledge, this is the first documented case of a supratentorial hemangioblastoma in a Filipino with vHL.

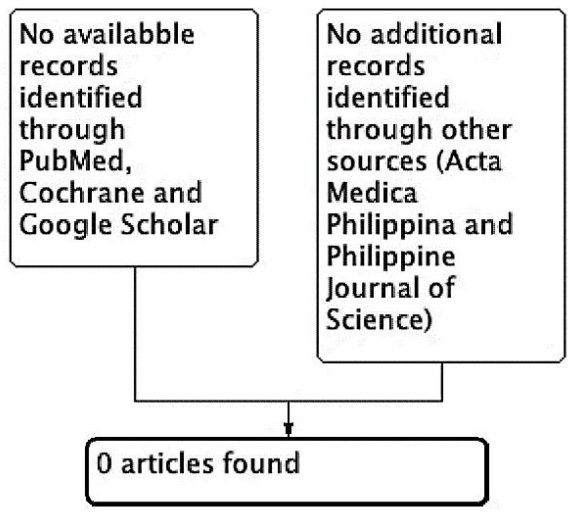

Figure 5: Database search strategy according to PRISMA guidelines.

Furthermore, as of the time of writing, there is no available local data on the prevalence or incidence of vHL syndrome in the Philippines. Diagnosis of this disease condition is challenging as it involves a high degree of suspicion and performance of several diagnostic tests which can be physically and financially taxing to the patient. This case is one of the very few reported in the country. Von Hippel-Lindau syndrome is a result of the mutation of the vHL gene causing loss of the functional protein leading to decreased degradation of the hypoxia-inducible factor (HIF). HIF, a transcription factor, plays a role in the expression of protumorigenic molecules such as the vascular endothelial growth factor
[2]. Diagnosis of vHL is achieved clinically when a patient presents with a positive family history and at least one typical vHL tumor. These include hemangioblastoma in the CNS, renal cell carcinoma (RCC), and pheochromocytoma. However, while vHL is recognized as a familial genetic condition, about $20 \%$ of patients are de novo cases. Presence of multiple tumors is therefore required for diagnosis in said instances [3, 4].

This patient has no known familial history of vHL syndrome nor any of the typical extracranial tumors associated with the disease entity. Despite this, extensive workup was done following high clinical suspicion for this syndrome. He is eventually documented to have mass lesions in the bilateral retina and the central nervous system suspicious for hemangioblastomas. Literature shows that about $20-43 \%$ of cases of hemangioblastomas is associated with vHL disease [3]. This incidence increases to up to $60 \%$ in those with supratentorially located tumors [5]. In addition, the patient is diagnosed with renal cell carcinoma which can be seen in 30-70\% of cases of vHL disease. The presence of these tumors is enough to fulfill the criteria for clinical diagnosis of a de novo vHL syndrome: i) more than one hemangioblastoma in the CNS or retina; ii) single hemangioblastoma in CNS or retina plus a visceral complication, i.e., renal cell cancer [4]. Imaging modalities play a key role in the screening and subsequent clinical diagnosis of this syndrome. However, genetic testing to detect germline mutation in the vHL gene best establishes the diagnosis especially in individuals without known family history of the disease [2]. Moreover, positive confirmation by DNA testing can provide insight on decisions regarding testing of asymptomatic family members. Our patient is still for genetic testing as an index case and if the results detect a germline mutation, his family members are primed to undergo the test as well.

A study by Peyre $e$ t al. showcased 409 patients with vHL disease in the VHL Study Group database in France from 1981-2008, but they only found $13(3.2 \%)$ patients with 18 supratentorial hemangioblastoma, where 4 patients had multiple tumors. Twelve patients underwent VHL genetic analysis, however, there was no association noted between specific germline VHL mutations and supratentorial hemangioblastomas. The hemangioblastomas were mainly located in the temporal lobe with most frequent clinical presentations were seizures, intracranial hypertension, and visual loss, and 14 of these tumors 
exhibited tumoral growth in their subsequent imaging. Two of them died from the syndrome or progression of supratentorial hemangioblastoma [6]. Our patient is still alive and presently undergoing oral systemic chemotherapy.

At present, there is no universal treatment for von-Hippel Lindau syndrome. Management of the disease still varies on a case-to-case basis and depends on the overall clinical picture of the individual [2,3, 7]. For this patient, treatment for the advanced renal cell carcinoma has been administered, beginning with nephrectomy then proceeding to systemic chemotherapy. He would have to undergo close follow ups and regular surveillance not only to monitor disease progression of renal cell carcinoma, but also to detect new growths related to vHL syndrome. Surveillance, especially when started at a younger age, is attributed to improving survival rates [8]. Studies show that among patients with vHL, most of the deaths (as high as $95.6 \%$ ) are related to their syndrome [8]. Renal carcinoma and complications of CNS hemangioblastoma such as bleeding are the primary causes of their demise [7, 8]. These two conditions are predictors of poorer overall survival, and both are present in this case. The patient is subsequently appraised of his fair prognosis and has decided to undergo treatment primarily for renal cancer.

In conclusion, this case highlights the importance of maintaining a high index of suspicion to prompt search for other associated disease features in any patient presenting with a typical vHL lesion. Delay in recognition and diagnosis of vHL syndrome can lead to increased morbidity and mortality from serious complications such as advanced renal carcinoma. The lack of familial history should not preclude further investigation of its presence.

\section{Acknowledgement}

The authors thank their colleagues from the Nephrology, Pulmonology, Cardiology, Urology and Medical Oncology services for their assistance and inputs on this case. They also would like to thank Dr. Erickson F. Torio for his additional inputs on this case.

\section{REFERENCES}

1. National Organization for Rare Disorders (2019) Von HippelLindau Disease.

2. Hes FJ, Höppener JW, Luijt RBvd, Lips CJ (2005) Von HippelLindau Disease. Hered Cancer Clin Pract 3: 171-178. [Crossref]

3. Varshney N, Kebede AA, Owusu Dapaah H, Lather J, Kaushik M et al. (2017) A Review of Von Hippel-Lindau Syndrome. J Kidney Cancer VHL 4: 20-29. [Crossref]

4. Wu P, Zhang N, Wang X, Ning X, Li T et al. (2012) Family history of von Hippel-Lindau disease was uncommon in Chinese patients: suggesting the higher frequency of de novo mutations in VHL gene in these patients. J Hum Genet 57: 238-243. [Crossref]

5. Mills SA, Oh MC, Rutkowski MJ, Sughrue ME, Barani IJ et al. (2012) Supratentorial hemangioblastoma: clinical features, prognosis, and predictive value of location for von Hippel-Lindau disease. Neuro Oncol 14: 1097-1104. [Crossref]

6. Peyre M, David P, Van Effenterre R, François P, Thys M (2010) Natural history of supratentorial hemangioblastomas in von HippelLindau disease. Neurosurgery 67: 577-587. [Crossref]

7. Binderup ML, Jensen AM, Budtz Jørgensen E, Bisgaard ML (2017) Survival and causes of death in patients with von Hippel-Lindau disease. J Med Genet 54: 11-18. [Crossref]

8. Zhou B, Wang J, Liu S, Peng X, Hong B et al. (2019) Hemangioblastoma Instead of Renal Cell Carcinoma Plays a Major Role in the Unfavorable Overall Survival of Von Hippel-Lindau Disease Patients. Front Oncol 9: 1037. [Crossref] 